\title{
超快速和二维液相色谱手性分离
}

\author{
许瑶康经武* \\ (中国科学院上海有机化学研究所 生命有机化学国家重点实验室 上海 200032)
}

\begin{abstract}
摘要 虽然以多糖类手性固定相为标志的手性分离技术已经趋于成熟, 但是复杂样品的手性分离仍然面临许多挑战. 为此, 近年来二维液相色谱手性分离得到了快速发展. 二维液相色谱需要第二维色谱分离要足够快, 因此又需要发展 超快速液相色谱分离技术. 超快速液相色谱和二维液相色谱的发展不仅仅提高了手性分离效率，同时也迎合了高通量 有机合成技术发展的需求. 总结了近年来超快速液相色谱和二维液相色谱在手性分析方面的研究进展, 重点讨论了超 快速液相色谱手性填料技术的发展和在解决复杂手性样品分析时的策略和应用, 最后对超快速和二维液相在高通量有 机合成中的应用前景做了展望.
\end{abstract}

关键词＼cjkstart超快速液相色谱; 二维液相色谱; 手性分离; 高通量分离

\section{Chiral Separation by Ultrafast and Two-Dimensional Liquid Chromatography}

\author{
$\mathrm{Xu}$, Yao Kang, Jingwu* \\ (State Key Laboratory of Bioorganic and Natural Products Chemistry, Shanghai Institute of Organic Chemistry, \\ Chinese Academy of Science, Shanghai 200032)
}

\begin{abstract}
Although the chiral separation techniques represented by the polysaccharides-based chiral stationary phases are almost matured, the chiral separation for complex samples remains a challenge. To this end, two dimensional liquid chromatography has been developed rapidly. Recently, to achieve the chiral separation with two dimensional liquid chromatoraphy, the development of the ultrafast liquid chromatography has caused considerable attention. The development of ultrafast liquid chromatography and two-dimensional liquid chromatography also caters to the development of high-throughput organic synthesis technology. In this review the recent progress in the chiral separation by the ultrafast and two-dimensional liquid chromatography is summarized. This review focuses on the progress in the techniques of the chiral packing materials for ultrafast chromatography, the chiral separation strategy and its application in complex samples. Finally, the application perspective of ultra-fast and two-dimensional liquid phase in high-throughput organic synthesis techniques is prospected.

Keywords ultrafast liquid chromatography; two-dimensional liquid chromatography; chiral separation; high-throughput separation
\end{abstract}

由于手性药物的对映体往往具有不同的药理和毒 理作用, 药物监管部门对手性药物的研发做出严格的规 定: 要求含手性中心的药物的所有对映体都需要进行单 独的药理和毒理评价 ${ }^{[1]}$. 尽管以多糖类手性固定相为代 表的液相色谱手性分析技术已经趋于成熟, 但是在分析 带有多个手性中心药物的立体异构体杂质和降解产物, 以及对生物样品中手性药物代谢产物的分离分析依然 是挑战性的工作. 这是由于手性固定相的分离选择性非
常窄, 仅能够实现对映体分离而无法进行非对映异构体 的分离 ${ }^{[2-3]}$. 这导致非对映异构体常常与生物样品中的 内源性物质共流出.二维液相色谱(2D-LC)是解决这一 问题的最佳选择. 在过去的十多年中, 人们发展了各种 二维液相色谱技术用于复杂样品的手性分离 ${ }^{[4]}$. 构建成 功的二维色谱的关键问题是第二维分离要足够快. 在过 去的 10 年里出现的超快速液相色谱不仅极大地提高了 分析速度, 实现了高通量的分离分析 ${ }^{[5-6]}$, 也为二维液相

\footnotetext{
* Corresponding author. E-mail: jingwu.kang@sioc.ac.cn

Received May 18, 2020; revised June 29, 2020; published online July 15, 2020.

Dedicated to the 40th anniversary of Chinese Journal of Organic Chemistry.

Project supported by the National Natural Science Foundation of China (Nos. 21775158, 21375140, 21175146).

国家自然科学基金(Nos. 21775158, 21375140, 21175146)资助项目.
} 
色谱的发展奠定了基础 ${ }^{[7-11]}$. 实际上这些技术的进步与 有机化学的发展有着密切的联系. 近年来为了提高新药 的研发速度, 制药界开始发展高通量的有机合成技术以 加快发现新的有机化学反应和优化合成条件的速 度 ${ }^{[12-13]}$, 如果涉及到高通量的不对称催化和不对称合成 方法, 就需要快速的手性分离分析技术与之相适应.

本文总结了近 10 年来在超快速和二维液相色谱在 手性分析中取得的研究进展, 重点介绍了超快速液相色 谱手性填料的发展和在解决复杂手性样品分析上的策 略以及应用. 最后对超快速和二维液相在高通量有机合 成中的应用前景做了展望.

\section{1 超快速液相色谱}

在过去的 30 年里, 手性分离技术的进步 ${ }^{[14-19]}$ 为不 对称合成、不对称催化以及手性药物的发展奠定了基 础 $^{[6,20]}$. 液相色谱作为手性分析的重要工具, 从 1980 年 代的 20 40 min, 到 2000 年代的 5 10 min, 直至最近 实现了亚分钟的超快速分离 ${ }^{[5-6]}$. 超快速液相色谱手性 分离的发展有望进一步提高有机化学家在研究不对称 催化和手性药物合成方面的效率.

快速的手性分离得益于液相色谱填料技术的进步. 2015 年, Armstrong 等 ${ }^{[21]}$ 将替考拉宁和万古霉素键合到 $1.9 \mu \mathrm{m}$ 窄粒径分布的全多孔硅胶填料上, 实现了亚分钟 的快速手性分离. 这种填充柱的背压比宽粒径分布的硅 胶材料低约 $80 \%$, 可减少摩擦生热, 允许色谱柱在高流 速使用. 作者将该技术用于多种手性化合物的分离分
析，包括不同类型的氨基酸， $\beta$-受体阻滞剂和合成药物 重要的杂环化合物(例如噁唑烷酮)等, 均得到了基线分 离. 这种键合大环糖肽的亚 $2 \mu \mathrm{m}$ 手性填料可用于包括 反相、正相和极性有机相的分离模式, 而且在不同模式 之间交替使用后，其性能没有任何下降. Ismail 等 ${ }^{[22]}$ 采 用与 Armstrong 等不同的键合化学, 在亚 $2 \mu \mathrm{m}$ 全多孔硅 胶填料上制备了一种带有烷基和带电铵基的替考拉宁 手性固定相，获得了极高的对映体分离效率. 作者在短 柱 $(2 \mathrm{~cm})$ 和超短柱 $(1 \mathrm{~cm})$ 上，对 $\mathrm{N}$ 端保护氨基酸、 $\alpha$-芳基 酸、除草剂以及消炎药进行手性分析，可得到基线分离, 分析时间约为 $1 \mathrm{~min}$.

2006 年推出了第二代表面多孔硅胶填料 ${ }^{[23]}$ ，也被 称为核壳填料. 这一类填料是利用纳米技术，在硅胶实 核周围堆积 $0.5 \mu \mathrm{m}$ 的多孔壳形成直径 $2.7 \mu \mathrm{m}$ 的球形颗 粒，多孔区域占据了填料总体积的约四分之三. 第二代 核壳填料在色谱柱展现了突破性的分离性能，柱效类似 于 $1.7 \mu \mathrm{m}$ 球形全多孔填料, 但是柱压却明显降低 [24-28].

Lindner 等 ${ }^{[29]}$ 早在 2011 年就首次报道了手性表面多 孔填料的应用. 他们在 $2.7 \mu \mathrm{m}$ 的表面多孔填料上键合 金鸡纳碱，形成阴离子交换手性固定相，用于氨基酸衍 生物的手性分析. 作者结合高灵敏度的 SRM 质谱检测, 实现了一次运行下对人体尿液样品中 16 种氨基酸的 $L-$ 对映体的手性分离鉴定. Chankvetadze 等 ${ }^{[30]}$ 报道了在表 面多孔填料表面涂覆三(4-氯-3-甲基苄基氨基甲酸酯)纤 维素，实现了高效的手性分析. 随后，他们 ${ }^{[31]}$ 利用涂覆

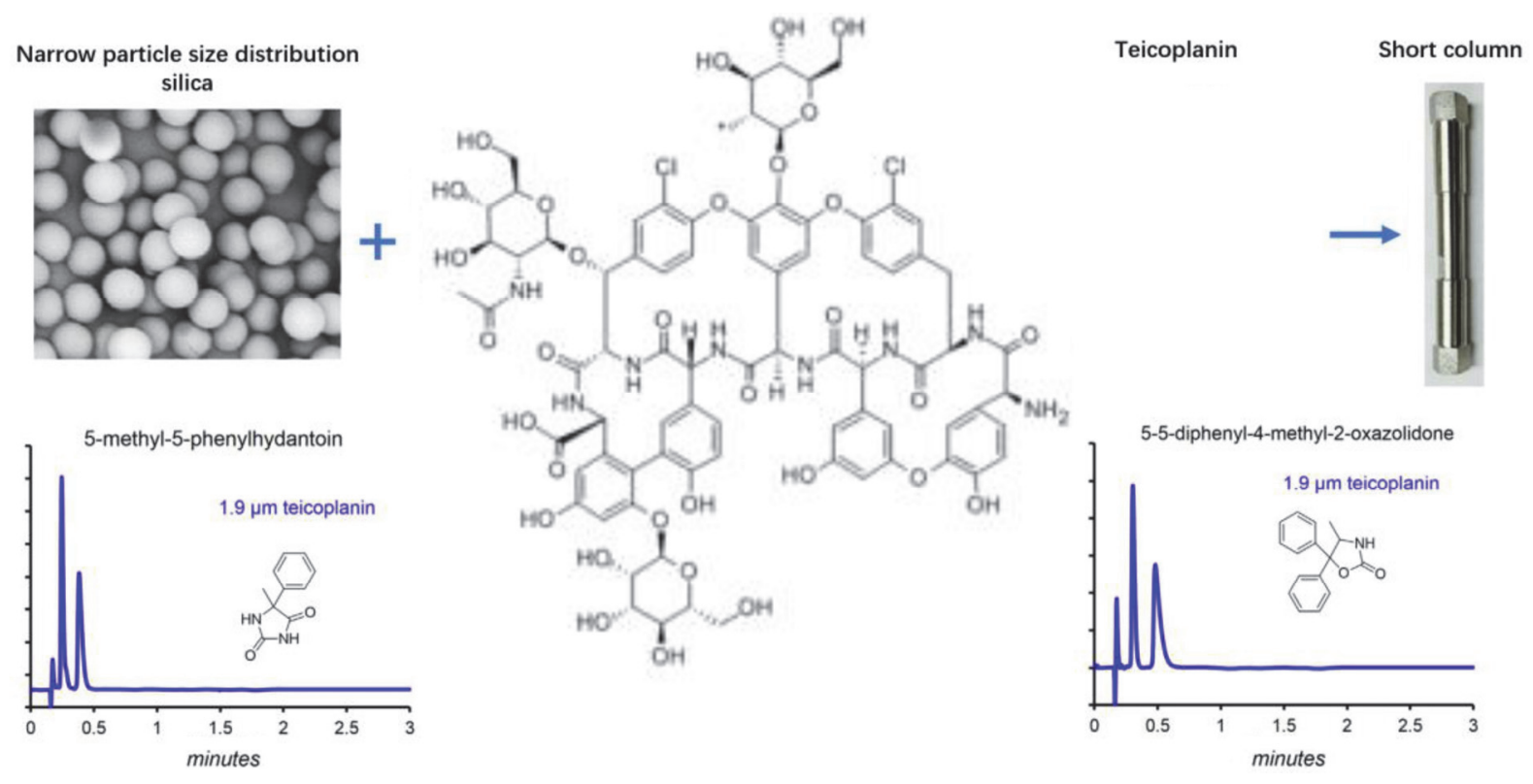

图 1 大环糖肽键合的亚 $2 \mu \mathrm{m}$ 硅胶填料高效手性固定相结合短柱可以实现超快分离

Figure 1 High efficiency chiral stationary phase in which chiral macrocyclic glycopeptides bonded on sub-2 $\mu \mathrm{m}$ silica and short columns allowed ultrafast separations 


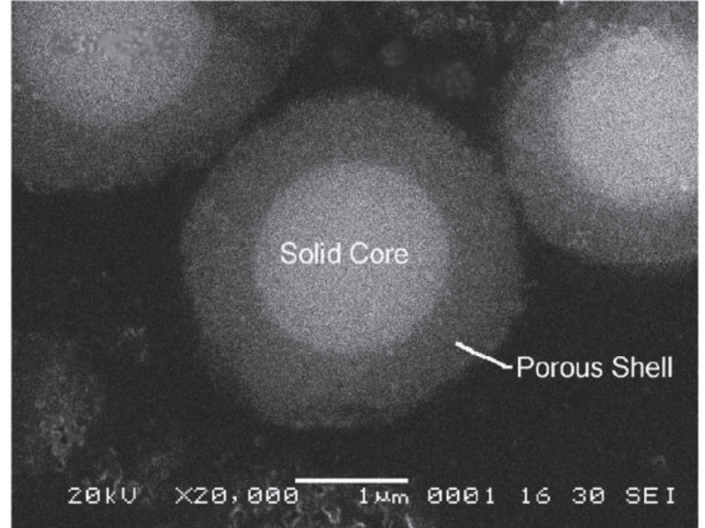

图 $22.7 \mu \mathrm{m}$ 核壳型硅胶填料的电镜图

Figure 2 Structure of $2.7 \mu \mathrm{m}$ core-shell silica packing material under TEM

直链淀粉三酯的表面多孔填料对安息香、曲格列酮碱以 及依托唑啉进行了超快速手性分离.

手性填料的改进还包括增强固相键合性能 ${ }^{[32-33]}$ 、拓 宽孔径 ${ }^{[34-35]}$ 、更广泛的键合相选择 ${ }^{[36-37]}$ 等. Armstrong 等 对表面多孔填料在高效、高通量的对映体手性分离上进 行了比较全面系统的评价. 他们利用 $2.7 \mu \mathrm{m}$ 表面多孔 填料制备了各种类型的手性固定相 ${ }^{[38]}$, 包括基于异丙 氨基甲酸酯衍生的环果聚糖的刷型手性固定相, 以及 $\beta$ 环糊精和大环抗生素(特别是替考拉宁、替考拉宁糖和 万古霉素)等键合手性固定相, 并对 BINAM 等 6 种对映 体实现了 $10 \sim 40 \mathrm{~s}$ 的超快速手性分离. 在所有色谱模式 中, 表面多孔填料在色谱动力学性能上都表现出了独特 的优越性. 作者还研究了 $20 \mathrm{~s}$ 的 $(R, S)$-BINAM 的手性分 离过程中, 检测器采样率和响应时间对 6 种对映体的分 离效率、分辨率和基线噪声的影响. 为了保证超高速液 相色谱方法足够的数据采样、检测器采样率和检测器响 应时间对于快速洗脱分析物和高效分离变得越来越重 要. 在某些情况下, 由于探测器的设置, 峰的形状、峰的 宽度和基线噪声可能会有很大的变化, 影响了对映体的 分离. 作者通过调整这些数据采集参数, 两个对映体之 间的分辨率可提高 10 倍. 随着采样率的增加和耦合时 间常数的减小, 当采样率达到 $80 \mathrm{~Hz}$ 左右时, 分离参数 有了很大的改善. 为了在进行超快分离时保持较高的效 率和良好的分辨率, 检测器耦合采样频率大于 $40 \mathrm{~Hz}$, 响应时间应小于 $0.13 \mathrm{~s}$.

Armstrong 等 ${ }^{[39]}$ 将手性表面多孔填料装填在 $0.5 \mathrm{~cm}$ 的超短柱中分离不同类型的化合物. 通过减少柱外体积 和实验条件的优化, 在不同的键合固定相(奎宁和替考 拉宁) 以及不同的色谱模式下成功实现了亚秒级的手性 分离. 高效液相色谱一质谱联用技术(HPLC-MS)已成为 药物分析领域中最主要的技术之一. 液质联用技术对复 杂生物基质中药物分子及其代谢物的质谱检测具有更

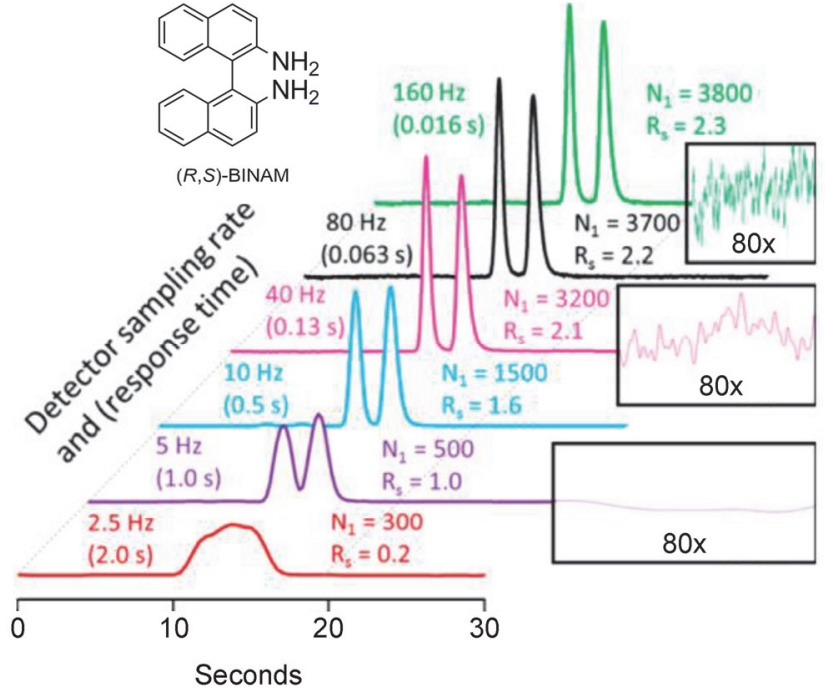

图 3 BINAM 对映体在不同数据采集速率和检测器时间常数 下的快速手性分离

Figure 3 Chiral separation of 1,1'-bi(2-naphthylamine) (BIN$\mathrm{AM})$ enantiomers at various data acquisition rates and detector time constants

高的选择性，可以避免更复杂的分离或者样品纯化过 程. Armstrong 等 ${ }^{[00]}$ 将基于 $2.7 \mu \mathrm{m}$ 表面多孔填料的万古 霊素手性固定相用于 22 种手性药物的 HPLC-MS 分离 分析. 他们的工作取得了高效的分离结果，同时成功分 离并鉴定了外消旋西酞普兰对映体的降解产物.

\section{2 超临界流体色谱}

超临界流体色谱(SFC)采用超临界 $\mathrm{CO}_{2}$ 作为色谱流 动相, 通过添加甲醇或异丙醇改性剂调节流动相的洗脱 强度 ${ }^{[41]}$. 由于超临界 $\mathrm{CO}_{2}$ 的粘度接近气体, 分析物具有 较高的扩散系数和较低的传质阻力, 并且在较高的流速 下依然能够保持较低的柱压力. 因此 SFC 可以使用较高 流速, 进一步减少峰展宽. SFC 的压力是普通液相色谱 的五分之一至三分之一, 所以相当于可以增加 3 到 5 倍 的流速，这意味着更快的运行时间、柱平衡和高通量. 在所有的色谱分析方法中, 无论在分离分析和制备分离 上, SFC 都是最有效的手性分离方法 ${ }^{[42]}$. 近年来 SFC 在 手性分离领域的优势已得到制药界的认可. 无论是在药 物发现过程中获得纯对映体, 还是在临床试验阶段取得 大量的原料药物, 制备 SFC 法都是手性化合物分离的首 选技术 ${ }^{[43]}$.

手性超临界流体色谱因其高速度和高效率而被用 作二维液相色谱法的第二维. 在分析规模上, 主要是改 进了 SFC 分析仪器性能和固定相, 特别是采用更小粒径 的手性固定相填料使得手性分离条件篮选过程大大缩 短. Biba 等 ${ }^{[4]}$ 研究比较了 $5 \mu \mathrm{m}$ 以及 $3 \mu \mathrm{m}$ 直径填料的填 充柱用于 SFC 快速手性分离, 结果显示小填料填充的色 
谱柱能够实现更快、更有效的分离. 实验采用恒定的背 压 $(20.0 \mathrm{MPa})$, 使用 $10 \%$ 的甲醇改进剂和 $40{ }^{\circ} \mathrm{C}$ 的温度. 作者在 $3 \mu \mathrm{m}$ 填料填充柱上使用优化的 SFC 方法分析了 华法林和羟基华法林的复杂手性混合物体系, 成功在 8 $\min$ 内分离出所有 12 种组分. Armstrong 等 ${ }^{[21]}$ 将 $1.9 \mu \mathrm{m}$ 全多孔硅胶填料键合大环糖肽作为手性固定相, 通过 SFC 对多种手性化合物进行分析, 得到了亚分钟的高效 手性分离. 他们 ${ }^{[45]}$ 将叔丁基氨基甲酰化奎宁与 $2.7 \mu \mathrm{m}$ 表面多孔填料结合, 制备了两种新型的阴离子交换柱, 建立了高效的手性 SFC 固定相. 新柱的性能和保留参数 比基于 $5 \mu \mathrm{m}$ 全多孔填料的手性柱效率提高了 3 倍. 该 技术对于多种 $\mathrm{N}$ 端保护氨基酸对映体实现了亚秒时间 的超快分离. Berger ${ }^{[46]}$ 利用亚 $2 \mu \mathrm{m}$ 填料进行 SFC 手性拆 分, 得到了高效的分离结果. 二苯乙烯氧化物对映体在 $1.8 \mu \mathrm{m}$ 硅胶手性柱上分离, 塔板高度低至 1.93. 他们根 据改性剂浓度、压力和温度的影响来进一步研究该手性 固定相的动力学性能. 经过条件优化后, 该 SFC 方法可 以对华法林、氟比洛芬、布洛芬等药物实现高效的手性 分离. 国内在 $\mathrm{SFC}$ 手性药物分离上也得到了很多进展, 陈小明等 ${ }^{[47]}$ 以多糖手性固定相为基础, 采用 SFC 成功 拆分了 11 种手性化合物. 他们比较了 6 种手性色谱柱对 这些手性化合物的手性识别, 均可以在 $10 \mathrm{~min}$ 内得到良 好的分离结果.

除了色谱柱外, 还可以在微流控芯片上实现超快速 的手性分离. Heiland 等 ${ }^{[48]}$ 报道了一种可以同时控制压 力和温度的微流控平台, 用于超快速的液相分离分析. 该装置可以产生高度规则对称的峰形. 采用双光子激发 荧光检测技术, 可以实现快速手性分离, 分离速度低于 $20 \mathrm{~s}$.

除了制药界, SFC 还被用于农药、法医物证、天然 产品和化妆品中的手性分析. 目前, 约 $30 \%$ 的农药含有 一个或多个手性中心. 虽然已经明确立体异构体的生物 活性存在显著差异, 在大多数情况下仍使用消旋混合 物. 近年来, 手性 SFC 被广泛用于分析食品、烟草和土 壤中的手性农药的残留. 张文华等 ${ }^{[49]}$ 采用多糖类手性 固定相的 SFC 法, 实现了对 6 种三唑类农药 16 种对映 体的高效分离, 并测定了这些农药在黄瓜中的残留. 李 中皓等 ${ }^{[50]}$ 采用 SFC-串联质谱法对烟草和土壤中的氟齐 弗丁对映体进行测定. 方法以 $\mathrm{CO}_{2}$ 和甲醇为流动相, 对 柱温、自动背压调节压力和改性溶剂进行了优化, 得到 了最佳的分离效率, 在 $4 \mathrm{~min}$ 内实现基线分离. 在此条 件下, 目标对映体的回收率为 $82.8 \% \sim 99.5 \% .2018$ 年, 董丰收等 ${ }^{[51]}$ 采用 SFC-串联质谱联用技术, 快速分析了 芬布康唑及其手性代谢物在水果、蔬菜、谷物和土壤中 的残留. 方法使用直链淀粉三酷涂覆的手性柱于 $4 \mathrm{~min}$
内实现了 6 个立体异构体的基线分离. 该方法采用柱后 补偿溶剂 $(0.1 \%$ 甲酸/甲醇) 优化电离效率和灵敏度. 罗逢 健等 ${ }^{[52]}$ 利用 SFC-四极杆飞行时间质谱联用技术，建立 了茶叶、苹果、葡萄中二唑对映体的手性分离及残留的 分析方法. 他们在涂覆了直链淀粉三酯的手性柱上将对 映体进行手性分离. SFC 手性分离方法简便、可靠，可用 于苹果、葡萄、茶叶样品中二唑类农药对映体的残留分 析和测定.

\section{3 二维液相色谱}

二维液相色谱是将两根以上的色谱柱通过一个接 口(interface)串联起来组成的分离系统. 二维色谱分离 必须满足两个条件: 两个柱的分离选择性互为正交; 已 经在第一维得到分离的样品不能在第二维混合. 二维液 相色谱的接口一般是采用两位的 6,8 或 10 通切换阀. 按 色谱柱的切换方式, 二维色谱分离系统可以分为中心切 割(Heart-cutting)和全二维(Comprehensive)液相色谱 ${ }^{[53]}$. 中心切割二维液相色谱用 LC-LC 表示, 只将第一维分 离中感兴趣的组分转移到第二维色谱柱进行进一步的 分离. 中心切割二维液相色谱方法又可以分为单次中心 切割和多次中心切割(mLC-LC). 多中心切割法允许将 第一维中的多个馏分依次转移到第二维中, 被广泛用于 解决结构非常近似的化合物的分离, 包括药物相关杂 质、异构体和降解产物 ${ }^{[54]}$. 全二维液相色谱用 $\mathrm{LC} \times \mathrm{LC}$ 表示, 从第一维分离的所有样品组份都要转移到第二维 分离柱. 标准的全二维液相色谱的接口是一个带有多个 样品收集环的两位 10 通切换阀, 样品依次进入第二维 色谱柱进行分析. 只有当第二维色谱完成上一个样品的 分离后, 才能将下一个样品环中的馏分引入到第二维色 谱柱中. 因此, 全二维色谱要求第二维色谱的分离要非 常快.

在手性分析时, 二维分离常采用非手性-手性色谱 柱的排布顺序 ${ }^{[55]}$. 在过去的十多年中, 人们发展了各种 二维液相色谱技术用于复杂样品的手性分离 ${ }^{[4,56-61]}$. 近 年来, 手性超临界流体色谱(SFC)因其高速度和高效率 而常被用作二维液相色谱的第二维. 在大多数情况下, 反相液相色谱(RPLC)和 SFC 组成的二维液相系统可以 满足复杂样品中对映选择性的分离需求 ${ }^{[62]}$. Venkatramani 等 ${ }^{[63]}$ 报道了 RPLC-SFC 中心切割二维液相色谱法, 同时进行非手性和手性分析. RPLC 和 SFC 由一个两位 8 通切换阀和小体积的 $\mathrm{C} 18$ 捕集柱组成的接口偶联. 从第 一维反相柱上洗脱的馏分被小体积 C18 捕集柱捕集后 聚焦浓缩, 然后注入第二维的手性 SFC 柱上进行分离.

表面多孔填料和超高压液相色谱技术的出现, 为二 维液相色谱在制药及生物领域的快速手性分析奠定了 


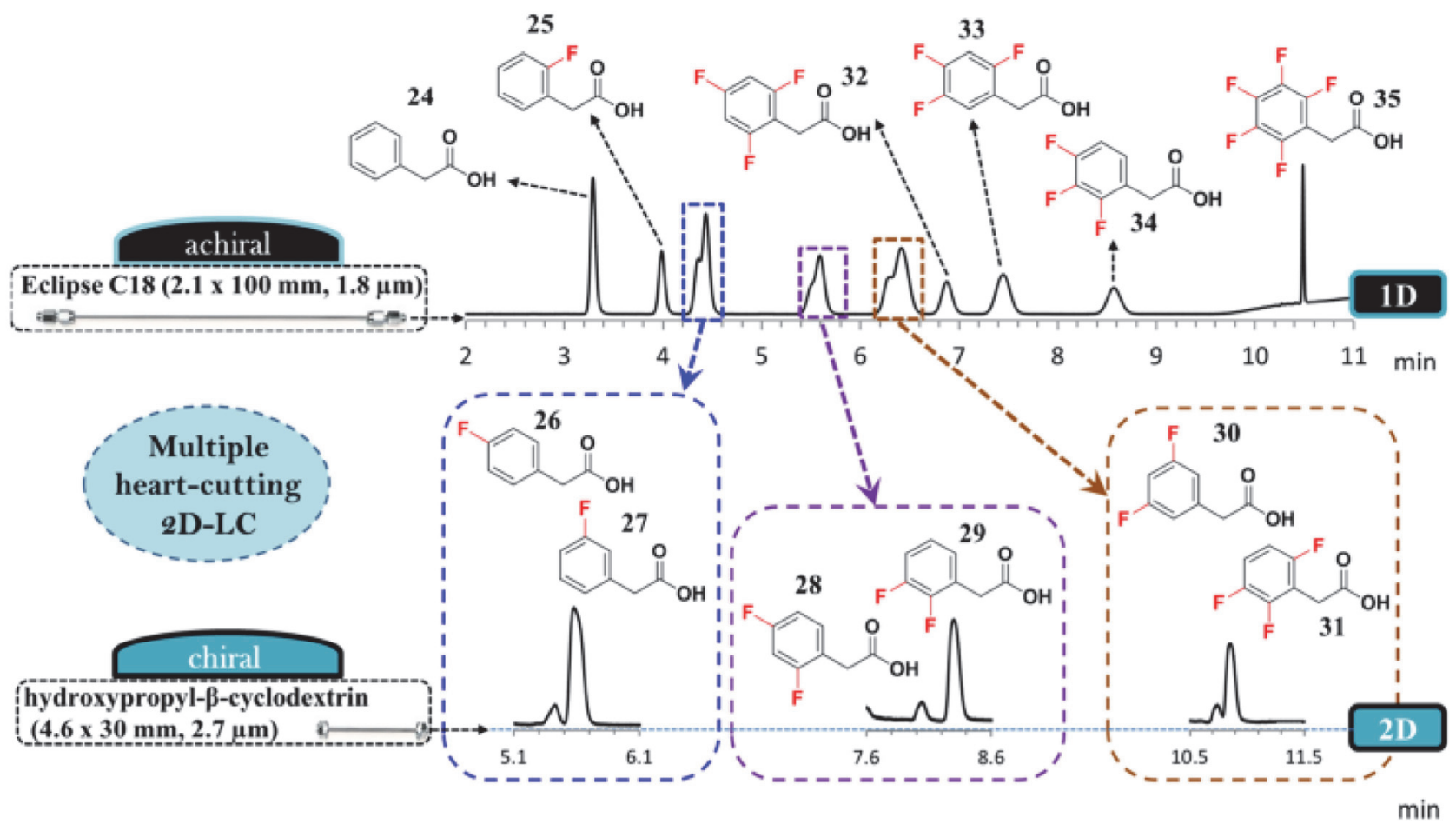

图 4 多次中心切割二维液相色谱法分离氟苯乙酸异构体混合物

Figure 4 Multiple heart-cutting 2D-LC method for separation of complex mixture of fluorophenylacetic acid isomers

基础. Barhate 等 ${ }^{[64]}$ 报道了在线手性-手性色谱柱结合的 全二维色谱法用于复杂样品的快速手性分析. 作者使用 超快手性分析柱作为第二维，对抗丙型肝炎蛋白酶抑制 剂研发过程中一个重要的合成中间体的所有立体异构 体进行了手性分离. 在 $0.1 \%$ 磷酸/乙腈的洗脱液条件下, 结果呈现优秀的选择性、峰形和可重复性, 证明了超快 手性反相色谱作为全二维液相色谱第二维的强大功能.

Bester 等 ${ }^{[65]}$ 发展了非手性-手性色谱柱结合的中心 切割二维高效液相色谱分离方法, 用于生物样品中六溴 环十二烷(HBCD)异构体对映选择性的测定. 异构体在 第一维的常规反相柱上分离后转移到第二维的手性柱 上. 分离过程在两个独立的色谱部分进行：一个用于 $\alpha-\mathrm{HBCD}$ 分析, 另一个用于 $\beta$-HBCD 和 $\gamma$-HBCD 分析, 实现了完全不受干扰的高效手性拆分. 作者还将此方法 应用于沙鳗油、剑鸥、环斑海豹等样品的手性分析中.

2019 年, Woiwode 等 ${ }^{[66]}$ 报道了手性-手性色谱柱结 合的全二维液相色谱法, 用于肽和蛋白水解样品的氨基 酸手性分析. 他们使用了两种手性选择性正交的手性固 定相, 将叔丁基氨基甲氧基奎宁 $(t-\mathrm{BuCQN})$ 和奎尼丁 $(t$-BuCQD) 衍生物键合在表面多孔硅胶填料, 使二维流 速比一维流速快 60 倍, 从而实现在线全二维色谱的快 速手性分析. 在二维色谱图中, 非手性组分和杂质峰在 二维分离空间的对角线上排列, 从而从目标对映体的色 谱空间中去除, 避免干扰; 相应的对映体在二维色谱图 上有交叉峰. 作者还将该方法应用于治疗性肽类药物格
拉米啶和杆菌肽的手性分析. 由于所有氨基酸的洗脱顺 序与芴甲氧羰基氨基酸衍生物一致，所以相同构型的所 有对映异构体都在对角线上或下，因此可以通过二维色 谱图直观反映肽段中氨基酸的构型. Woiwode 等 ${ }^{[60]}$ 在另 一份工作中利用在线全自动二维液相分离技术，在多次 中心切割和选择性全二维模式下, 实现了分离和量化所 有 20 种蛋白氨基酸的 $D$ 和 $L$ 型对映体.

当面对带有多个手性中心药物的分离问题时，由于 立体异构体的数量增长为 $2^{n}$, 情况会更加具有挑战性. 一个例子是泊沙康唑, 它是一种广谱的三唑抗真菌剂, 用于治疗由孢粉和镰刀菌引起的侵袭性曲霉病、念珠菌 病和真菌感染 ${ }^{[67]}$. 它有四个手性中心，可产生 16 个潜 在的立体异构体. 但是, 通过汇聚合成途径, 活性药物 成分中会引入 11 种相关的立体异构杂质. 因此，需要一 种监控活性药物成分中立体异构体的方法. 我们课题 组 ${ }^{[68]}$ 发展了多级中心切割二维液相色谱法, 用于分离 泊沙康唑的 12 个立体异构体杂质. 在优化流动相体系 的基础上，我们在三种键合型多糖手性固定色谱柱构成 的二维液相色谱系统上, 构建了多个中心切割的手性一 手性二维液相色谱系统. 采用对立体异构体保留相对较 弱的纤维素三酯涂覆的 IB 手性柱作为第一维, 对立体 异构体保留较强的直链淀粉三酯涂覆以及纤维素三酯 涂覆的 IF3 和 IC 手性柱作为第二维，从而将第一维分离 中的目标峰转移到二维色谱柱中, 避免了样品稀释的同 时也减小了样品切割时间对二维分离的影响. 该系统一 

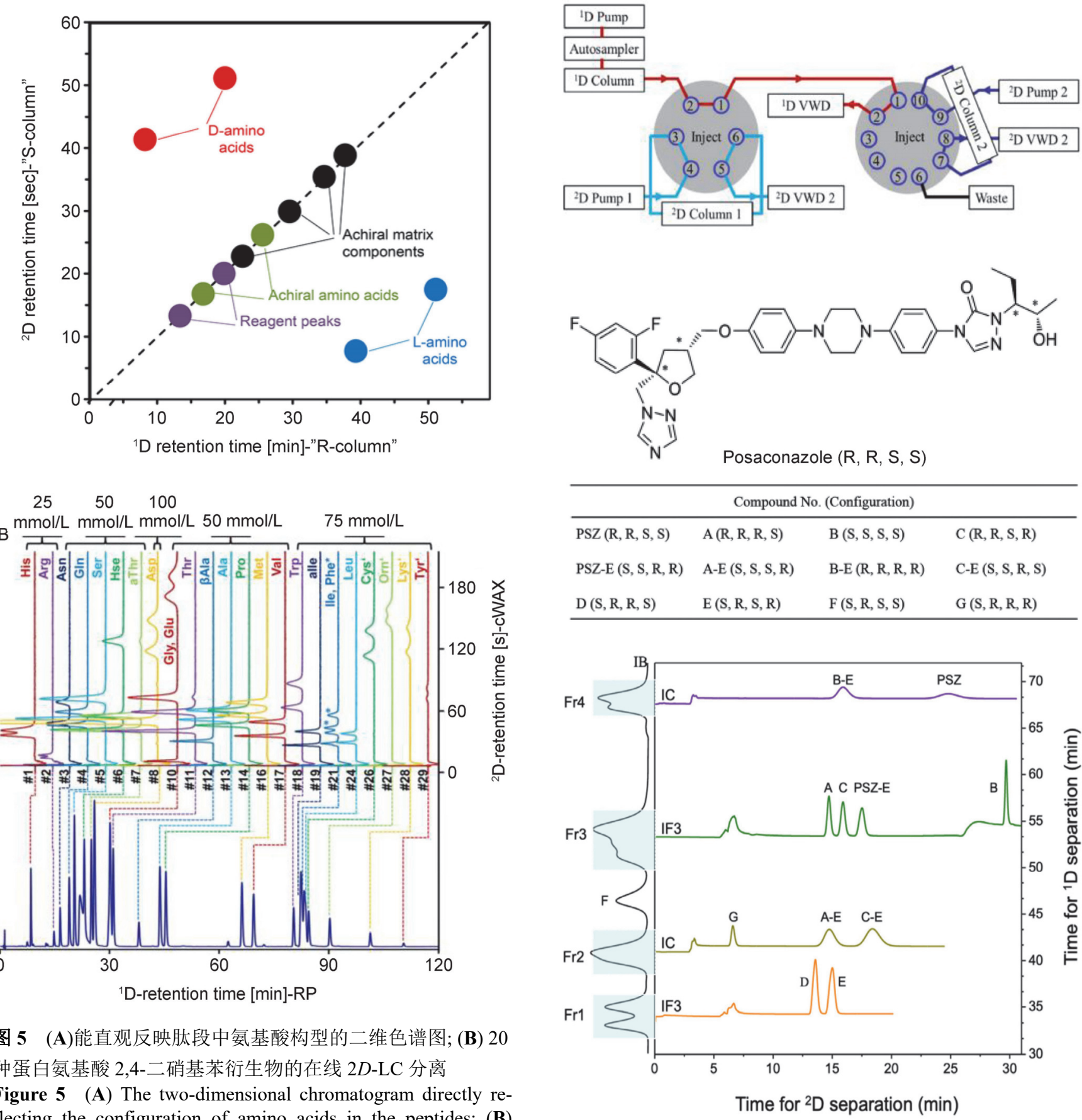

图 5 (A)能直观反映肽段中氨基酸构型的二维色谱图; (B) 20 种蛋白氨基酸 2,4-二硝基苯衍生物的在线 $2 D-\mathrm{LC}$ 分离

Figure 5 (A) The two-dimensional chromatogram directly reflecting the configuration of amino acids in the peptides; (B) Online 2D-LC separation of 2,4-dinitrobenzene (DNP) derivatives of all 20 proteinogenic amino acids

次运行即可同时实现 12 个立体异构体的手性分离. 该 方法可应用于监测泊沙康唑制备过程中的立体异构体, 对其它具有多个不对称中心的药物的手性分析具有一 定的方法学参考价值.

\section{4 结论与展望}

近年来, 超快速和二维液相色谱手性分离技术得到 了快速发展, 已经成为手性药物研究的有利工具, 也为 复杂手性样品的分析提供了解决方案. 可以预计, 这些

图 6 (A)中心切割二维液相色谱系统原理图; (B) 12 种泊沙康 唑相关立体异构体的化学结构及其绝对构型; (C)中心切割二

维色谱法分离 12 种泊沙康唑相关立体异构体

Figure 6 (A) Schematic diagram of the multiple heart-cutting 2D-HPLC system; (B) the chemical structures of the 12 posaconazole related stereoisomers and their absolute configuration; (C) chromatograms for the separation of 12 posaconazole related stereoisomers on heart cutting 2D-LC system

技术也将为不对称性合成和不对称性催化研究提供更 好的工具. 近年来发展起来的高通量有机合成技术和合 成条件篮选技术已经得到广泛的关注，但是，对高通量 有机合成产生的样品分析依然是一个难题 ${ }^{[12]}$, 尤其是 
高通量不对称催化和不对称合成产物的分析将是超快 速手性分析需要关注的一个重要问题. 高通量合成技术 采用微升级的反应体积, 由于有机试剂对分离存在很大 的影响, 样品预处理也是快速手性分离需要解决的难 题. 相信随着越来越多的人开展这方面的研究, 会不断 推出更好的手性固定相, 新的仪器, 新分析方法和新的 样品预处理技术. 未来超快速和二维液相色谱手性分离 将极大地提高手性科学研究的效率.

\section{References}

[1] De Camp, W. H. J. Pharm. Biomed. Anal. 1993, 11, 1167.

[2] Maier, N. M.; Franco, P.; Lindner, W. J. Chromatogr. A 2001, 906, 3.

[3] Hegade, R. S.; Lynen, F. J. Chromatogr. A 2019, 1586, 116.

[4] Pirok, B. W. J.; Stoll, D. R.; Schoenmakers, P. J. Anal. Chem. 2019, 91, 240.

[5] Lorenz, H.; Seidel-Morgenstern, A. Angew. Chem., Int. Ed. 2014, $53,1218$.

[6] Regalado, E. L.; Kozlowski, M. C.; Curto, J. M.; Ritter, T.; Campbell, M. G.; Mazzotti, A. R.; Hamper, B. C.; Spilling, C. D.; Mannino, M. P.; Wan, L.; Yu, J. Q.; Liu, J.; Welch, C. J. Org. Biomol. Chem. 2014, 12, 2161.

[7] Major, E. R. LC GC Europe 2012, 25, 1.

[8] Mazzeo, J. R.; Neue, U. D.; Kele, M.; Plumb, R. S. Anal. Chem. 2005, 77, $460 \mathrm{~A}$.

[9] Blue, L. E.; Franklin, E. G.; Godinho, J. M.; Grinias, J. P.; Grinias, K. M.; Lunn, D. B.; Moore, S. M. J. Chromatogr. A 2017, 1523, 17.

[10] MacNair, J. E.; Lewis, K. C.; Jorgenson, J. W. Anal. Chem. 1997, $69,983$.

[11] Jorgenson, J. W. Annu. Rev. Anal. Chem. 2010, 3, 129.

[12] Perera, D.; Tucker, J. W.; Brahmbhatt, S.; Helal, C. J.; Chong, A.; Farrell, W.; Richardson, P.; Sach, N. W. Science 2018, 359, 429.

[13] Robbins, D. W.; Hartwig, J. F. Science 2011, 333, 1423.

[14] Barhate, C. L.; Wahab, M. F.; Tognarelli, D. J.; Berger, T. A.; Armstrong, D. W. Anal. Chem. 2016, $88,8664$.

[15] Ismail, O. H.; Ciogli, A.; Villani, C.; De Martino, M.; Pierini, M.; Cavazzini, A.; Bell, D. S.; Gasparrini, F. J. Chromatogr. A 2016, $1427,55$.

[16] Patel, D. C.; Breitbach, Z. S.; Wahab, M. F.; Barhate, C. L.; Armstrong, D. W. Anal. Chem. 2015, 87, 9137.

[17] Regalado, E. L.; Welch, C. J. J. Sep. Sci. 2015, 38, 2826.

[18] Wahab, M. F.; Wimalasinghe, R. M.; Wang, Y.; Barhate, C. L.; Patel, D. C.; Armstrong, D. W. Anal. Chem. 2016, 88, 8821.

[19] Welch, C. J.; Regalado, E. L. J. Sep. Sci. 2014, 37, 2552.

[20] Kotoni, D.; Ciogli, A.; Molinaro, C.; D'Acquarica, I.; Kocergin, J.; Szczerba, T.; Ritchie, H.; Villani, C.; Gasparrini, F. Anal. Chem. 2012, 84, 6805 .

[21] Barhate, C. L.; Wahab, M. F.; Breitbach, Z. S.; Bell, D. S.; Armstrong, D. W. Anal. Chim. Acta 2015, 898, 128.

[22] Ismail, O. H.; Ciogli, A.; Villani, C.; De Martino, M.; Pierini, M.; Cavazzini, A.; Bell, D. S.; Gasparrini, F. J. Chromatogr. A 2016, $1427,55$.

[23] Kirkland, J. J.; Langlois, T. J.; DeStefano, J. J. Am. Lab. 2007, 39, 18.

[24] Gritti, F.; Leonardis, I.; Abia, J.; Guiochon, G. J. Chromatogr. A 2010, 1217, 3819.

[25] Guiochon, G.; Gritti, F. J. Chromatogr. A 2011, 1218, 1915.

[26] Hayes, R.; Ahmed, A.; Edge, T.; Zhang, H. J. Chromatogr. A 2014, $1357,36$.

[27] Jandera, P.; Hájek, T.; Staňková, M. Anal. Bioanal. Chem. 2015, 407, 139.

[28] Tanaka, N.; McCalley, D. V. Anal. Chem. 2016, 88, 279.

[29] Reischl, R. J.; Hartmanova, L.; Carrozzo, M.; Huszar, M.; Fruhauf,
P.; Lindner, W. J. Chromatogr. A 2011, 1218, 8379.

[30] Lomsadze, K.; Jibuti, G.; Farkas, T.; Chankvetadze, B. J. Chromatogr. A 2012, 1234, 50

[31] Kharaishvili, Q.; Jibuti, G.; Farkas, T.; Chankvetadze, B. J. Chromatogr. A 2016, 1467, 163.

[32] Berger, T. A. J. Chromatogr. A 2017, 1510, 82.

[33] Bezhitashvili, L.; Bardavelidze, A.; Mskhiladze, A.; Gumustas, M.; Ozkan, S. A.; Volonterio, A.; Farkas, T.; Chankvetadze, B. J. Chromatogr. A 2018, 1571, 132.

[34] D'Orazio, G.; Kakava, R.; Volonterio, A.; Fanali, S.; Chankvetadze, B. Electrophoresis 2017, 38, 1932.

[35] Mao, X. J.; Li, J.; Liu, D.; Qiao, T.; Ma, L.; Sun, X.; Xu, L.; Shi, Z. G. Talanta 2018, 178, 583 .

[36] Kucerova, G.; Kalikova, K.; Tesarova, E. Chirality 2017, 29, 239.

[37] Roy, D.; Armstrong, D. W. J. Chromatogr. A 2019, 1605.

[38] Patel, D. C.; Breitbach, Z. S.; Wahab, M. F.; Barhate, C. L.; Armstrong, D. W. Anal. Chem. 2015, 87, 9137.

[39] Wahab, M. F.; Wimalasinghe, R. M.; Wang, Y.; Barhate, C. L.; Patel, D. C.; Armstrong, D. W. Anal. Chem. 2016, 88, 8821.

[40] Guo, H.; Wahab, M. F.; Berthod, A.; Armstrong, D. W. J. Pharm. Anal. 2018, 8, 324

[41] Franzini, R.; Ciogli, A.; Gasparrini, F.; Ismail, O. H.; Villani, C. In Chiral Analysis, Elsevier Science, Rome, Italy, 2018, pp. $607 \sim 629$.

[42] West, C. TrAC, Trends Anal. Chem. 2019, 120.

[43] Speybrouck, D.; Lipka, E. J. Chromatogr. A 2016, 1467, 33.

[44] Biba, M.; Regalado, E. L.; Wu, N.; Welch, C. J. J. Chromatogr. A 2014, 1363, 250 .

[45] Patel, D. C.; Breitbach, Z. S.; Yu, J.; Nguyen, K. A.; Armstrong, D. W. Anal. Chim. Acta 2017, 963, 164.

[46] Berger, T. A. J. Chromatogr. A 2016, 1459, 136.

[47] Li, D. Y.; Wu, X.; Hao, F. L.; Yang, Y.; Chen, X. M. Chin. J. Chromatogr. 2016, 34, 80 (in Chinese). (李冬艳, 吴锡, 郝芳丽, 杨洋, 陈小明, 色谱, 2016, 34, 80.)

[48] Heiland, J. J.; Geissler, D.; Piendl, S. K.; Warias, R.; Belder, D. Anal. Chem. 2019, 91, 6134.

[49] Zhang, W. H.; Xie, W.; Hou, J. B.; Chen, Q. K.; Li, S. M.; Zhu, Z. L.; Zou, X. Q; Xu, D. M. Chin. J. Chromatogr. 2019 37, 1356 (in Chinese).

(张文华，谢文，侯建波，陈钦可，李淑敏，祝泽龙，邹学权，徐 敦明，色谱, 2019, 37, 1356.)

[50] Yang, F.; Tang, G.; Liu, S.; Fan, Z.; Wang, Y.; Deng, H.; Bian, Z.; Li, Z. Chirality 2019, 31, 353.

[51] Tao, Y.; Zheng, Z.; Yu, Y.; Xu, J.; Liu, X.; Wu, X.; Dong, F.; Zheng, Y. Food Chem. 2018, 241, 32.

[52] Zhang, X.; Zhao, Y.; Cui, X.; Wang, X.; Shen, H.; Chen, Z.; Huang, C.; Meruva, N.; Zhou, L.; Wang, F.; Wu, L.; Luo, F. J. Chromatogr. A 2018, 1581-1582, 144.

[53] León-González, M. E.; Rosales-Conrado, N.; Pérez-Arribas, L. V.; Guillén-Casla, V. Biomed. Chromatogr. 2014, 28, 59.

[54] D'Atri, V.; Fekete, S.; Clarke, A.; Veuthey, J. L.; Guillarme, D. Anal. Chem. 2019, 91, 210.

[55] Barhate, C. L.; Joyce, L. A.; Makarov, A. A.; Zawatzky, K.; Bernardoni, F.; Schafer, W. A.; Armstrong, D. W.; Welch, C. J.; Regalado, E. L. Chem. Commun. 2017, 53, 509.

[56] Barhate, C. L.; Regalado, E. L.; Contrella, N. D.; Lee, J.; Jo, J.; Makarov, A. A.; Armstrong, D. W.; Welch, C. J. Anal. Chem. 2017, $89,3545$.

[57] Ianni, F.; Sardella, R.; Lisanti, A.; Gioiello, A.; Cenci Goga, B. T.; Lindner, W.; Natalini, B. J. Pharm. Biomed. Anal. 2015, 116, 40.

[58] Rao, R. N.; Kumar, K. N.; Shinde, D. D. J. Pharm. Biomed. Anal. 2010, 52, 398.

[59] Venkatramani, C. J.; Al-Sayah, M.; Li, G.; Goel, M.; Girotti, J.; Zang, L.; Wigman, L.; Yehl, P.; Chetwyn, N. Talanta 2016, 148, 548 .

[60] Woiwode, U.; Neubauer, S.; Lindner, W.; Buckenmaier, S.; Lämmerhofer, M. J. Chromatogr. A 2018, 1562, 69.

[61] Woiwode, U.; Reischl, R. J.; Buckenmaier, S.; Lindner, W.; Lämmerhofer, M. Anal. Chem. 2018, 90, 7963. 
[62] Iguiniz, M.; Corbel, E.; Roques, N.; Heinisch, S. J. Pharm. Biomed. Anal. 2018, 159, 237.

[63] Venkatramani, C. J.; Al-Sayah, M.; Li, G.; Goel, M.; Girotti, J.; Zang, L.; Wigman, L.; Yehl, P.; Chetwyn, N. Talanta 2016, 148, 548.

[64] Barhate, C. L.; Regalado, E. L.; Contrella, N. D.; Lee, J.; Jo, J.; Makarov, A. A.; Armstrong, D. W.; Welch, C. J. Anal. Chem. 2017,
$89,3545$.

[65] Bester, K.; Vorkamp, K. Anal Bioanal Chem 2013, 405, 6519.

[66] Woiwode, U.; Reischl, R. J.; Buckenmaier, S.; Lindner, W.; Lammerhofer, M. Anal. Chem. 2018, 90, 7963.

[67] Schiller, D. S.; Fung, H. B. Clin. Ther. 2007, 29, 1862.

[68] Xu, F.; Xu, Y.; Liu, G.; Zhang, M.; Qiang, S.; Kang, J. J Chromatogr. A 2020, 460845. 